\section{Intracellular pathogens within alveolar macrophages in a patient with HIV infection: diagnostic challenge}

\author{
Takashi Shinha, ${ }^{1}$ Olga Badem² \\ 'Department of Pathology, Microbiology \\ and Immunology, Vanderbilt University \\ Medical Center, Nashville, IN; \\ 2Department of Medicine, Wyckoff \\ Heights Medical Center, Brooklyn, NY, \\ USA
}

\begin{abstract}
In HIV-infected individuals, macrophages, the key defense effector cells, manifest defective activity in their interactions with a wide variety of opportunistic pathogens, including fungi and protozoa. Understanding the morphological characteristics of intracellular opportunistic pathogens in addition to their pathogenesis is of critical importance to provide optimal therapy, thereby decreasing morbidity and mortality in HIV-infected patients. We herein present a case of disseminated histoplasmosis confused with disseminated visceral leishmaniasis in an HIV-infected individual from Guyana who developed intracellular organisms within alveolar macrophages.
\end{abstract}

\section{Introduction}

Human immunodeficiency virus (HIV)infected individuals are at increased risk for various opportunistic infections. Histoplasma typically manifest as disseminated histoplasmosis in HIV-infected patients with advanced stages of immunosuppression. Leishmania can also cause systemic manifestations as a form of visceral leishmaniasis (VL) in HIV-infected patients, though it is less familiar to clinicians in non-endemic areas for leishmaniasis, including the United States. Importantly, both Histoplasma and Leishmania have the capability of surviving in defective macrophages associated with impaired cell-mediated immunity in the presence of HIV infection. Thus, morphologically differentiating those two pathogens is paramount in the presence of similar systemic manifestations. We describe an HIV-infected individual who developed disseminated histoplasmosis that was initially thought as disseminated VL. Intracellular organisms within alveolar macrophages from bronchial alveolar lavage (BAL) were highly suspicious for amastigotes of Leishmania species. We also review other cases of HIV- infected patients co-infected with Leishmania to discuss atypical manifestations of VL in the presence of HIV infection.

\section{Case Report}

A 47 year-old man with a history of HIV infection was in his usual status until 14 days before presentation, when fever, malaise, and mild intermittent diarrhea developed. He also reported night sweats and unintentional weight loss of three pounds during that period. The patient was camping in Long Island in New York (USA) when his symptoms started. He recalled multiple mosquito bites during camping. Otherwise, he denied any respiratory, urinary or neurological symptoms. A diagnosis of HIV infection had been made four years before. Antiretroviral therapy was administered intermittently due to noncompliance. At the time of presentation, the patient was taking abacavir, emtricitabine, and ritonavir-boosted fosamprenavir for HIV and trimethoprim-sulfamethoxazole for prophylaxis. Past HIV-related opportunistic infections included oral candidiasis. He was born in Guyana and immigrated to the United States in 1984. The most recent travel to Guyana was five years prior to presentation. He worked in a public service position. He drank alcohol on occasions and never smoked tobacco or used illicit drugs. On physical examination, the patient appeared chronically ill and diaphoretic. The blood pressure was $122 / 89 \mathrm{~mm} \mathrm{Hg}$, pulse 116 beats per minute, temperature $102.2^{\circ} \mathrm{F}\left(39.0^{\circ} \mathrm{C}\right)$, respirations 20 breaths per minute, and oxygen saturation was $96 \%$ on room air. Oral thrush as well as multiple cervical lymphadenopathy was observed. His heart sounds were regular without murmurs. His lungs were clear to auscultation. No rash or edema was observed. The remainder of the physical examination was unremarkable.

Laboratory studies on admission revealed a white blood cell count of 1900 cells per cubic millimeter (ref 4500-10,800) with 90\% neutrophils (reference range 36-66), hematocrit of $36 \%$ (reference range 41-53) and platelets of 126,000 per cubic millimeter (ref 150,000$450,000)$. The serum level of sodium was 136 $\mathrm{mEq} / \mathrm{L}$ (ref 137-145), potassium $4.7 \mathrm{mEq} / \mathrm{L}$ (ref 3.5-5.1), urea nitrogen $22 \mathrm{mg} / \mathrm{dL}$ (ref 9-20), creatinine $1.7 \mathrm{mg} / \mathrm{dL}$ (ref 0.6-1.2), alanine aminotransferase $45 \mathrm{U} / \mathrm{L}$ (ref 13-69), aspartate aminotransferase $208 \mathrm{U} / \mathrm{L}$ (ref 15-46), alkaline phosphatase $86 \mathrm{U} / \mathrm{L}$ (ref 38-126), total bilirubin $0.3 \mathrm{mg} / \mathrm{dL}$ (ref $0.2-1.3$ ), lactate dehydrogenase $8977 \mathrm{U} / \mathrm{L}$ (ref 313-618), and lactic acid 2.0 $\mathrm{mmol} / \mathrm{L}$ (ref $0.7-2.1$ ). The partial thromboplastin time (PTT) was 30.4 seconds (ref 22.035.0) and INR was 1.2 (ref 0.8-1.2). The CD4+ T-lymphocyte cell count was 12 cells per cubic
Correspondence: Takashi Shinha, Department of Pathology, Microbiology and Immunology; TVC Suite 4514; 1301 Medical Center Drive, Nashville, TN 37232, USA.

Tel.: +1.615.343.1289 - Fax: +1.615.322.2504

E-mail: takashi.shinha@vanderbilt.edu

Key words: HIV, intracellular pathogens, alveolar macrophages, histoplasmosis, leishmaniasis.

Acknowledgements: the authors would thank Dr. Miguel Echevarria for the pathology review.

Contributions: TS and OB contributed to study conception; TS contributed to initial drafting of the manuscript.

Conflict of interests: the authors declare no potential conflict of interests.

Received for publication: 4 December 2014.

Revision received: 22 December 2014.

Accepted for publication: 30 December 2014.

This work is licensed under a Creative Commons Attribution NonCommercial 3.0 License (CC BYNC 3.0).

(C) Copyright T. Shinha and O. Badem, 2015 Licensee PAGEPress, Italy

Infectious Disease Reports 2015; 7:5747 doi:10.4081/idr.2015.5747

millimeter and the level of HIV nucleic acid by PCR was 55,000 copies/mL. A chest radiograph did not show active pulmonary lesions. A computed tomography (CT) of the chest without contrast enhancement showed bilateral multiple punctate nodules in the upper lung fields (Figure 1A).

The patient was started on cefepime, azithromycin, primaquine, clindamycin, and fluconazole on admission. He continued to have high fevers and five days after admission, he developed a sudden onset of hypoxia. There were no significant pulmonary findings in a repeat chest radiograph. Subsequently, he underwent bronchoscopy with BAL. Specimens were sent for bacterial, fungal, and acid-fast bacilli (AFB) staining. Cytological examination of BAL from the right middle lobe with Giemsa stain revealed alveolar macrophages containing numerous intracellular organisms (Figure 1B). Ciliated columnar cells and inflammatory cells were also present. The specimens from BAL were sent to the Centers for Disease Control and Prevention (CDC). The cultures and polymerase chain reaction (PCR) testing for Leishmania species were negative. Pathological examination of the bone marrow biopsy showed no malignant cells or granuloma. However, Grocott's methenamine silver (GMS) stain revealed numerous yeasts morphologically consistent with Histoplasma cap- 
sulatum (Figure 1C). The patient eventually recovered after a prolonged course of anti-fungal therapy with amphotericin followed by itraconazole.

\section{Discussion}

Disseminated histoplasmosis is recognized as an opportunistic infection in HIV infected patients. Macrophages and dendritic cells are the major effector cells in host resistance to various fungi, including Histoplasma. ${ }^{1}$ Defective macrophages associated with impaired cell-mediated immunity in the presence of HIV bind fewer yeast cells than cells from HIV-negative individuals and the capacity of macrophages to bind yeast cells decrease with more advanced immunosuppression. ${ }^{2}$ Unlike acute pulmonary histoplasmosis occurring in immunocompetent hosts, pulmonary involvement may not be prominent in disseminated histoplasmosis associated with HIV infection. As a result, chest radiographic findings of disseminated histoplasmosis in HIVinfected patients are varied and nonspecific. Conces et al. reported chest radiographic findings of 50 patients with AIDS, who developed disseminated histoplasmosis. Twenty-three patients demonstrated abnormal findings on chest X-ray, of which nodular opacities were the most common finding found in 10 patients. $^{3}$

Visceral leishmaniasis is also considered an opportunistic infection in HIV-infected patients particularly in areas endemic for leishmaniasis, such as southern European countries. ${ }^{4}$ Leishmania species have the capability of surviving intracellularly by various mechanisms. Leishmania release effectors that enhance macrophage phosphotyrosine phosphatase activity, ${ }^{5}$ and the surface lipophosphoglycan inhibits the macrophage oxidative burst by suppressing protein kinase C activity. ${ }^{6}$ Clinically, HIV-infected patients with VL may present with atypical clinical presentations. For instance, among 120 cases of VL reported by Pintado et al., of which 80 patients were co-infected with HIV, the HIV-coinfected patients had a lower frequency of splenomegaly than the HIV-negative individuals $(80.8 \%$ versus $97.4 \%) .{ }^{7} \mathrm{VL}$ in the presence of HIV infection may also affect uncommon sites, including the lungs. ${ }^{8}$ Various chest radiographic manifestations have been reported in HIV-infected patients co-infected with VL, including solitary pulmonary nodules and pleural effusion.9,10 Bronchioloalveolar adenoma associated with bronchiolitis obliterans and bilateral consolidative mass lesions with subsequent detection of Leishmania amastigotes inside the alveolar macrophages, were also reported. ${ }^{11,12}$ The patient was born in Guyana,
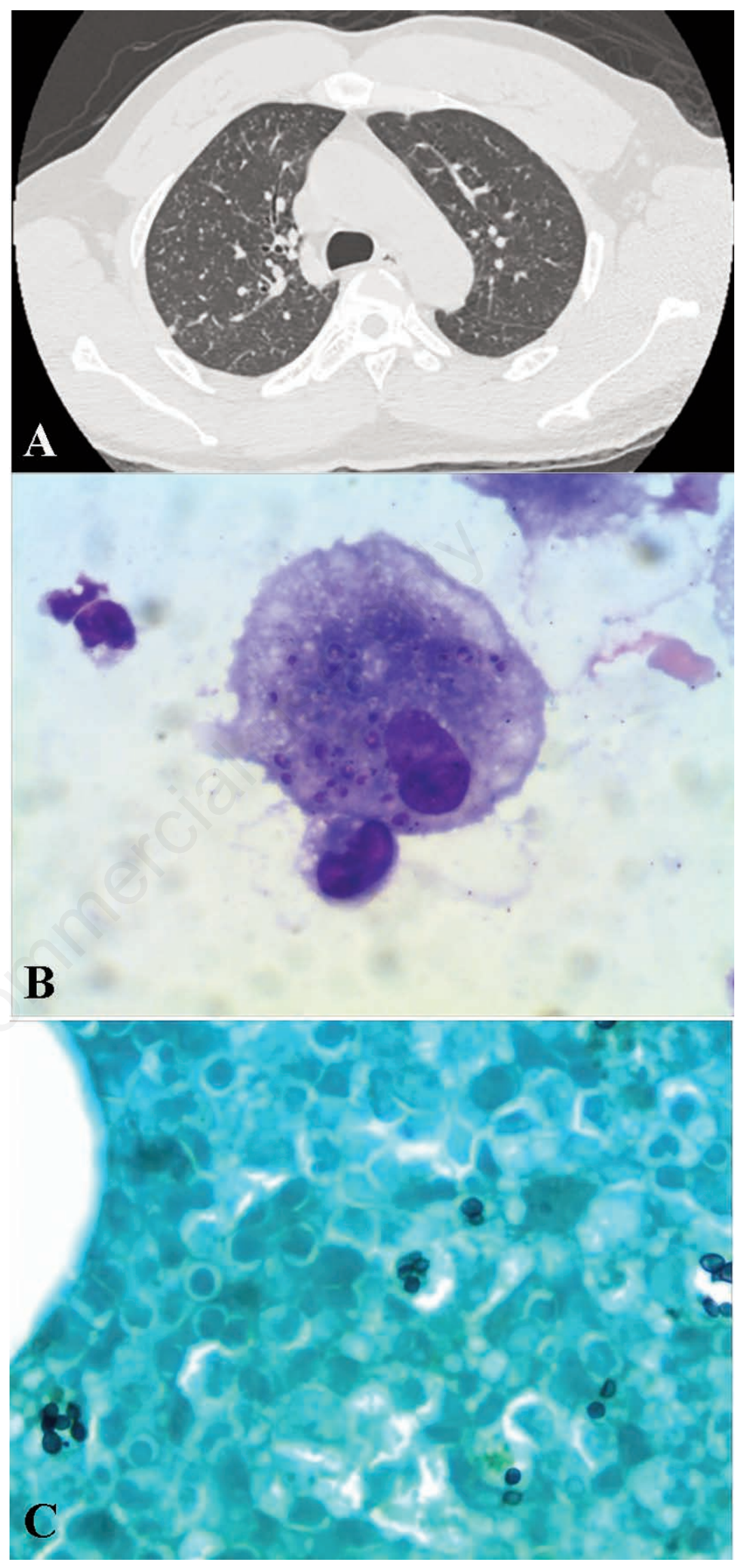

Figure 1. A) Chest computed tomography; B) Giemsa stain of bronchial alveolar lavage showing macrophages with intracellular organisms $(100 \times)$; C) Grocott's methenamine silver stain of bone marrow showing yeasts $(100 \times)$. 
where L. guyanensis is endemic particularly in its sylvatic or jungle areas. In general, L. guyanensis is the etiological agent for cutaneous leishmaniasis, and no cases of visceral leishmaniasis due to $L$. guyanensis have been reported in the literature in both HIV negative and HIV positive individuals. Although the patient was severely immunocompromized due to his underlying advanced stages of HIV infection, serology testing for Leishmania may have aided in the evaluation of prior acquisition of leishmaniasis.

Morphologically, Histoplasma and Leishmania may appear similar as round organisms surviving in macrophages, though they can be differentiated by visualizing the kinetoplasts in amastigotes of Leishmania. A kinetoplast is a network of DNA inside mitochondria that contains many copies of the mitochondrial genome. ${ }^{13}$ Kinetoplasts are found in kinetoplastids that are a group of flagellated protozoa. ${ }^{14}$ Because of the subtle distinguishing characteristics of kinetoplasts, identification of amastigotes within alveolar macrophages often requires experienced pathological skills particularly when organisms are sparse. Other pathogens, such as Toxoplasma, Pneumocystis, and Candida glabrata, may be confused with Histoplasma and/or Leishmania species. Intracellular yeasts of Histoplasma capsulatum is usually surrounded by a halo, though it may not be apparent in the presence of cell disruption. Intracellular bradyzoites of Toxoplasma gondii can form a pseudocyst inside the macrophage, and free extracellular Toxoplasma tachyzoites may also be seen from BAL. In conjunction with clinical presentations relevant to each suspicious pathogen, additional staining methods, including GMS, may aid in differentiation among these organisms. Of note, as far as we know, there is only one case report in the literature describing coexistence of VL and disseminated histoplasmosis in a patient with HIV infection. ${ }^{15}$

\section{Conclusions}

HIV-infected individuals are susceptible to opportunistic pathogens that can survive within macrophages in the presence of impaired cell-mediated immune function. Both Histoplasma and Leishmania can cause devastating disseminated infections in HIV-infected patients, particularly in those with more advanced stages of immunosuppression, thereby causing significant morbidity and mortality. Differentiating these two pathogens is of critical importance, however it may be challenging when they survive intracellularly as round structures in defective macrophages. Understanding characteristic morphologies of opportunistic pathogens in association with pathogenesis is paramount for clinicians who care for HIV-infected individuals.

\section{References}

1. Gildea LA, Ciraolo GM, Morris RE, Newman SL. Human dendritic cell activity against Histoplasma capsulatum is mediated via phagolysosomal fusion. Infect Immun 2005;73:6803-11.

2. Chaturvedi S, Frame P, Newman SL. Macrophages from human immunodeficiency virus-positive persons are defective in host defense against Histoplasma capsulatum. J Infect Dis 1995;171:320-7.

3. Conces DJ Jr, Stockberger SM, Tarver RD, Wheat LJ. Disseminated histoplasmosis in AIDS: findings on chest radiographs. AJR Am J Roentgenol 1993;160:15-9.

4. Alvar J, Cañavate C, Gutiérrez-Solar B, et al. Leishmania and human immunodeficiency virus coinfection: the first 10 years. Clin Microbiol Rev 1997;10:298-319.

5. Gomez MA, Olivier M. Proteases and phosphatases during Leishmania-macrophage interaction: paving the road for pathogenesis. Virulence 2010;1:314-8.
6. Descoteaux A, Matlashewski G, Turco SJ. Inhibition of macrophage protein kinase C-mediated protein phosphorylation by Leishmania donovani lipophosphoglycan. J Immunol 1992;149:3008-15.

7. Pintado V, Martín-Rabadán P, Rivera ML, et al. Visceral leishmaniasis in human immunodeficiency virus (HIV)-infected and non-HIV-infected patients. A comparative study. Medicine (Baltimore) 2001;80:54-73.

8. Rosenthal E, Marty P, del Giudice P, et al. HIV and Leishmania coinfection: a review of 91 cases with focus on atypical locations of Leishmania. Clin Infect Dis 2000;31:1093-5.

9. Casado JL, Cuesta C, Sanchez JA, Guerrero A. Solitary pulmonary nodule due to Leishmania in a patient with AIDS. Clin Infect Dis 1998;26:532-4.

10. Chenoweth CE, Singal S, Pearson RD, et al. Acquired immunodeficiency syndromerelated visceral leishmaniasis presenting in a pleural effusion. Chest 1993;103:6489.

11. Herrejon A, Cervera A, Maciá M, et al. [Bronchioloalveolar adenoma associated with bronchiolitis obliterans and leishmaniasis with lung involvement in acquired immunodeficiency syndrome]. Arch Bronconeumol 2005;41:233-5. [Article in Spanish].

12. Nigro L, Montineri A, La Rosa R, et al. Visceral leishmaniasis and HIV co-infection: a rare case of pulmonary and oral localization. Infez Med 2003;11:93-6.

13. Shapiro TA, Englund PT. The structure and replication of kinetoplast DNA. Annu Rev Microbiol 1995;49:117-43.

14. Stuart K, Brun R, Croft S, et al. Kinetoplastids: related protozoan pathogens, different diseases. J Clin Invest 2008;118:1301-10.

15. Manfredi R, Mazzoni A, Pileri S, et al. Simultaneous occurrence of visceral leishmaniasis and disseminated histoplasmosis in an Italian patient with HIV infection. Infection 1994;22:224-5. 